\title{
Characterization of heavily aluminized energetic material for explosive testing and chemical modelling
}

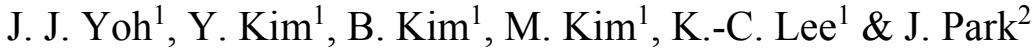 \\ ${ }^{I}$ Department of Mechanical \& Aerospace Engineering \\ Seoul National University, Korea \\ ${ }^{2}$ Agency for Defense Development, Korea
}

\begin{abstract}
The chemical response of energetic materials is analysed in terms of 1) the thermal decomposition under thermal stimulus and 2) the reactive flow upon the mechanical impact, both of which give rise to an exothermic thermal runaway or an explosion. The present study aims at building a set of chemical kinetics that can precisely model both thermal and impact initiation of a heavily aluminized cyclotrimethylene-trinitramine (RDX) which contains 35\% of aluminium. For a thermal decomposition model, the differential scanning calorimetry (DSC) measurement is used together with the Friedman isoconversional method for defining the frequency factor and activation energy in the form of Arrhenius rate law that are extracted from the evolution of product mass fraction. As for modelling the impact response, a series of unconfined rate stick data are used to construct the size effect curve which represents the relationship between detonation velocity and inverse radius of the sample. For validation of the modelled results, a cook-off test and a pressure chamber test are used to compare the predicted chemical response of the aluminized RDX that is either thermally or mechanically loaded.
\end{abstract}

Keywords: chemical kinetics, aluminium, energetic materials, $R D X, D S C$.

\section{Introduction}

The understanding of the response of propellants and high explosives is of broad interest to the energetic materials community for the purposes of safe handling, 
storage methods, and comprehension of reaction violence. In particular accurate kinetics are essential for predicting the chemical response of energetic materials.

Several approaches to determine the reaction profile of energetic materials have been proposed. The first one develops detailed mechanism that covers more than hundred reactions [1]. Most of such high energy materials have complex reaction profile which cannot be described by a single reaction step. Nevertheless, the kinetics composed of too many specific reactions are not suitable in view of computational chemistry for its exhaustively demanding computing time. Another approach assumes only a few reaction steps which have dominance on the global reactive response [2-5]. Furthermore, an enhancement to the isoconversional approach of Friedman [6] can provide the activation energy and frequency factor which vary with each degree of conversion during the progress of chemical reaction. This last approach has recently gained much attention, thanks to a noticeable progress in the integral method [7] and the availability on the kinetics analysis software [8].

To understand the thermal decomposition characteristics of energetic material, DSC is used [8-10]. The use of isoconversional method to extract the kinetics theoretically does not require any numerical fitting. Because the scheme is essentially a single step with a multiple set of Arrhenius parameters that evolve with the reaction progress, there is an obvious computational advantage over any multi-step kinetics.

As for modelling the chemical response of energetic materials under an impact loading, Lee and Tarver [11] or JWL++ (Jones-Wilkins-Lee++) [12] type approaches have been considered in general. Lee-Tarver considers the generation of hotspots and their effect during initiation. The model requires predetermination of parameters both empirically and ad hoc, making it difficult to be universal for generalized energetic material. The JWL ++ model offers simplification to [11] by ignoring the initiation step and using only the single growth step for detonating explosive. The methodology to determine model parameters are somewhat qualitative, and the reactive flow model would need to be recalibrated each time a new explosive is formulated. Once calibrated for one explosive, it does not manifest as to how to change the unknown parameters for other explosives.

In this paper, DSC experiment is used in building a thermal decomposition model, and rate stick experiment is performed to construct the ignition and growth rate model for the aluminized RDX. Then the models are validated through the cook-off experiment for thermal test and a pressure chamber test for shock initiation of the multi-purpose energetic material. The high concentration of aluminium within RDX, in the shock initiation test, calls for properly modelling two sequential reactions: the primary detonation of RDX proceeds the deflagration of aluminized powders ignited subsequently at a high pressure state of RDX product gases. Both thermal and shock test results confirm the applicability of the present set of kinetics to a general characterization of the energetic response of the target sample. 


\section{Thermal decomposition model using DSC data}

\subsection{Differential scanning calorimetry}

DSC experiments are carried out on Mettler Toledo DSC821e. The sample masses are in the range of $2.50-2.55 \mathrm{mg}$. The four heating rates of $0.5,1.0,2.0$ and $4.0^{\circ} \mathrm{C} / \mathrm{min}$ are used. The temperature range of $30-450^{\circ} \mathrm{C}$ is covered at all heating rates. For all DSC signals, positive going feature corresponds to exothermic process. The aluminum sealed closed pans are used: to endure the pressure generated by exothermic reaction of energetic materials, closed pans must be adopted for the present experiment. Since evaporation is faster than decomposition in the sample in an open pan DSC, one must consider the closed pan DSC in order to observe the exothermic chemical reaction.

\subsection{Kinetics calculation}

The reaction rate $d \lambda / d t$ and the mass fraction of product $\lambda(t)$ are obtained from Eqs (1) and (2).

$$
\begin{gathered}
\frac{d \lambda}{d t}=\frac{S(t)-B(t)}{\int_{t_{0}}^{t_{\text {end }}}[S(t)-B(t)] d t}, \quad \lambda(t)=\frac{\int_{t_{0}}^{t} S(t)-B(t) d t}{\int_{t_{0}}^{t_{\text {end }}}[S(t)-B(t)] d t}(0 \leq \lambda \leq 1) \\
B(t)=[1-\lambda(t)]\left(a_{1}+b_{1} t\right)+\lambda(t)\left(a_{2}+b_{2} t\right)
\end{gathered}
$$

Here $S(t)$ is DSC signal as a function of time $t . B(t)$ is called baseline which is a reference line for determining the magnitude of heat flow. The construction of baseline involves the superposition of tangents at each side of the exothermic signal peak. Each tangent is linked through the product mass fraction in a baseline function. The determination of baseline is conducted using AKTSThermokinetics Software [8]. The reaction rate at the time is instant heat flow divided by the summation of the released energy during the full chemical reaction process. The product mass fraction at the time is given by the summation of the released energy divided by the total released energy.

The DSC signals of heavily aluminized RDX are plotted in Fig. 1. In this figure, as heating rate increases, the temperature range during which the chemical reaction occurs becomes narrower, and the peak value of signal becomes higher. Also the reaction begins and also terminates at a higher temperature overall. From the DSC signals, we can see that the temperature range of the main reaction zone is $170-240^{\circ} \mathrm{C}$ for all heating rates. It means that the present DSC experiments cover only the reaction of RDX and the reaction of aluminum components are not considered because aluminum reaction begins at around $2200^{\circ} \mathrm{C}$. Consequently, kinetics extracted from DSC signals represent a thermal response of RDX component of the aluminized substance. 
From DSC signals, the heat of reaction can be calculated by Eq. (3).

$$
Q=\int_{t_{0}}^{t_{\text {end }}}[S(t)-B(t)] d t
$$

At each DSC signal in different heating rates, the heat of reaction $Q$ is calculated. The average value from four heating rates is used as heat of reaction of the target sample, and the biggest deviation from this experiment is $9.5 \%$. AKTS-Thermokinetics software provides guideline for reliably obtaining the heat of reaction, and such the biggest deviation below $10 \%$ is considered reliable. Under this reliability condition, the obtained heat of reaction for the aluminized RDX sample is $Q=1024.3 \mathrm{~J} / \mathrm{g}$.

As the DSC experiment is conducted for a specified heating rate, relationship between $d \lambda / d t$ and temperature can be obtained. Then isoconversional method is applied to DSC data with several heating rates, usually 4-5 heating rates, to extract the kinetics parameters [8-10].

In the Friedman isoconversional method, the reaction rate of materials is given in an Arrhenius form

$$
\frac{d \lambda}{d t_{\lambda}}=\left[A_{\lambda} f(\lambda)\right] \exp \left(\frac{-E_{\lambda}}{R T_{\lambda}\left(t_{\lambda}\right)}\right)
$$

Here $R, t_{\lambda}, T_{\lambda}, A_{\lambda}, E_{\lambda}$ are universal gas constant, time, temperature, frequency factor and activation energy at product mass fraction $\lambda$ respectively. $f(\lambda)$ is commonly used as a set of functions reflecting the reaction mechanism. In the present work, activation energy and frequency factor are calculated without the assumption of $f(\lambda)$. We take a logarithm of both sides of Eq. (4):

$$
\ln \frac{d \lambda}{d t_{\lambda}}=\ln \left[A_{\lambda} f(\lambda)\right]-\frac{E_{\lambda}}{R T_{\lambda}}
$$

In an Arrhenius plot, $-E_{\lambda} / R$ is slope and $\ln \left[\mathrm{A}_{\lambda} f(\lambda)\right]$ is the intercept with vertical axis $\ln [d \lambda / d t]$. The Friedman analysis of the aluminized RDX with four heating rates is presented in Fig. 2. Dotted line represents a $\lambda=0.95$ line that connects each heating rate curve. The slope and y-intercept of the dotted line represent the activation energy and the frequency factor at a corresponding $\lambda$,

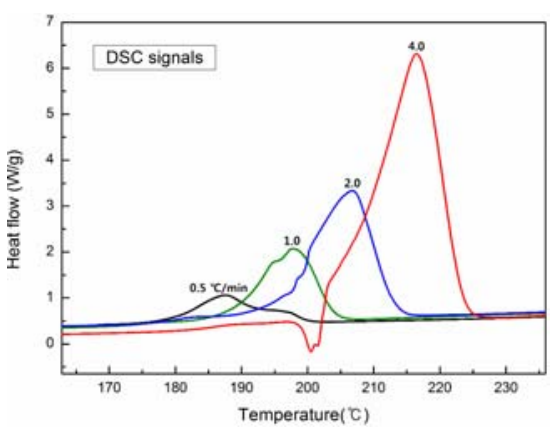

Figure 1: DSC signals.

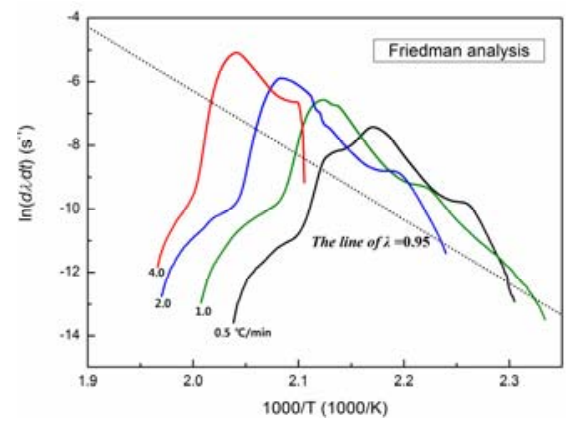

Figure 2: Friedman analysis. 
respectively. Thus, the comprehensive Arrhenius parameters for an entire process of reaction can be constructed where the progress, $\lambda$ varies from 0 to 1 . The obtained set of activation energy and frequency factor vary with $\lambda$ as depicted in Fig. 3. This implies that the extracted kinetics describe a full chemical reaction process elaborated through a set of Arrhenius parameters. One notes that the entire scheme is extracted rather theoretically without any artificial numerical fittings, which is an added advantage of the presented procedure.

\subsection{Kinetics validation}

The governing equations of constant volume reaction are summarized as in Eqs (6) and (7).

$$
\begin{gathered}
\frac{d T}{d t}=\dot{w} \\
\frac{d \lambda}{d t}=A_{\lambda} \exp \left(\frac{-E_{\lambda}}{R T}\right)
\end{gathered}
$$

where $\dot{w}$ is the heating rate. In the DSC experiment, the heat generated from reaction is emitted to the reference material immediately, and such the enthalpy of reaction is neglected. The Arrhenius parameters in Fig. 3 are used in the validation calculation. The comparisons of DSC experiment and simulation are plotted in Fig. 4. Here, a single step method as opposed to the present kinetics is considered at a heating rate of $4.0^{\circ} \mathrm{C} / \mathrm{min}$. The corresponding one-step reaction is given by

$$
\frac{d \lambda}{d t}=A_{0} \exp \left(\frac{-E_{0}}{R T}\right)
$$

where the values of $A_{0}=\exp (42)$ and $E_{0}=200 \mathrm{~kJ} / \mathrm{mol}$ are used. As it is evident from the comparison in Fig. 4, slope discontinuity is reproduced by the DSC based kinetics whereas single-step method cannot. The present kinetics extracted from the closed pan DSC require just two reaction parameters that evolve with time from initial to a final product state of the target sample. Thus with good accuracy, there is a definite advantage of the present scheme in the full scale hydrodynamic simulation of the thermally activated energetic material.

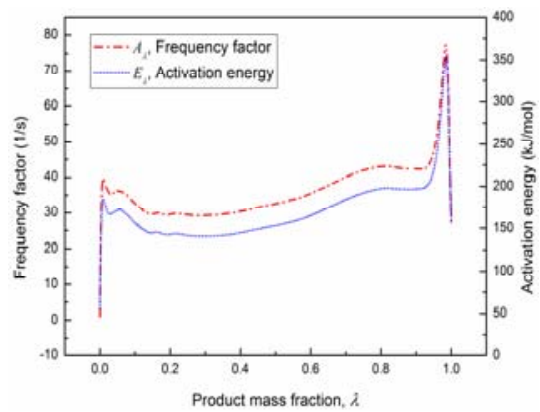

Figure 3: Frequency factor and activation energy.

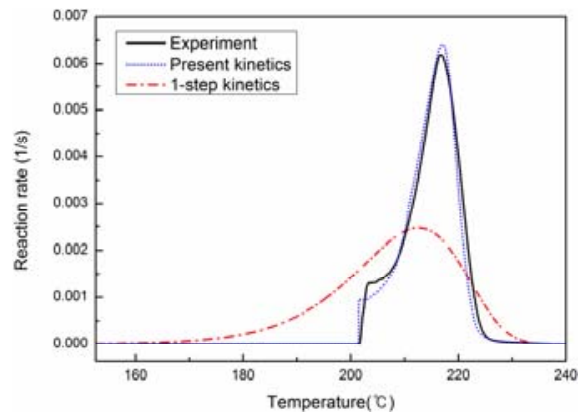

Figure 4: Rate of reaction compared at heating rate $4.0^{\circ} \mathrm{C} / \mathrm{min}$. 


\section{Impact initiation model using rate stick data}

The rate of product mass fraction consists of ignition and growth terms [13] such that

$$
\begin{aligned}
\frac{d \lambda}{d t}= & I(1-\lambda)^{b}(\eta-a)^{x} \quad, \eta=\frac{\rho}{\rho_{0}}-1 \\
& +G_{1}(1-\lambda)^{c} \lambda^{d} P^{y}+G_{2}(1-\lambda)^{e} \lambda^{g} P^{z}
\end{aligned}
$$

As before $\lambda$ is the burned mass fraction, and there are 12 constants $I, b, a, x, G_{l}, c$, $d, y, G_{2}, e, g, z$ to be determined. $P$ is the pressure, $t$ is time, $\rho_{0}$ and $\rho$ are the initial and current densities, respectively. The Mie-Gruneisen EOS [14] in Eq. (10) is for the unreacted solid, and the isentropic JWL EOS [15] in Eq. (12) is used for the reacted gaseous product.

$$
\begin{gathered}
P_{\text {unreacted }}=P_{H}+\Gamma \rho\left(e-e_{H}\right) \\
P_{\text {reacted }}=A e^{-R_{1}\left(\rho_{0} / \rho\right)}+B e^{-R_{2}\left(\rho_{0} / \rho\right)}+C\left(\rho_{0} / \rho\right)^{-(1+\omega)}
\end{gathered}
$$

Here $P_{H}, e_{H}$ are pressure and internal energy of a reference state that follows the Hugoniot curve, and $\Gamma$ is the Gruneisen gamma. $A, B, C, R_{1}, R_{2}$ are the material dependent JWL parameters with $\omega$ being the Gruneisen coefficient of Eq. (11). The standard mixture rule applies to the internal energy and volume ratio such that

$$
\begin{aligned}
& P_{\text {total }}=(1-\lambda) P_{\text {unreacted }}+\lambda P_{\text {reacted }} \\
& v_{\text {total }}=(1-\lambda) v_{\text {unreacted }}+\lambda v_{\text {reacted }} \\
& e_{\text {total }}=(1-\lambda) e_{\text {unreacted }}+\lambda e_{\text {reacted }}
\end{aligned}
$$

This type of Lee-Tarver model for reactive flow simulation comprises i) ignition term that represents formation of the hotspots by the rapid compression, and ii) first growth term that describes the effect of the propagation of the reacting waves in the substance and second growth term that represents completion due to a detonation transition. For determination of 12 unknowns above, a curve fitting method with optimization technique is required.

An alternative to these two limiting forms of the reactive flow equation that we adapt in this work can be casted into a following form [16] such that

$$
\frac{d \lambda}{d t}=I(1-\lambda) \eta^{a}+G(1-\lambda) P^{b}, \eta=\frac{\rho}{\rho_{0}}-1
$$

Here, the model resembles a full Lee-Tarver, while the initiation step is added to the JWL++. 4 unknown parameters having the major significance in view of detonation are kept, namely $I, a, G, b$, which is an advantage over the classical one. The initiation step requires defining the ignition constant, $I$, while the reactant depletion $(1-\lambda)$ is time-resolved in the governing equation with a compression $\eta=\rho / \rho_{0}-1$. The ignition of high explosive occurs by compression because of the shock wave propagation. Hotspots are formed in a shocked high explosive, leading to a void collapse. Any void or gas bubble that exists in 
high explosives may provide a potential site for local adiabatic compression that leads to the localized heating well beyond the activation energy for detonation.

The four parameters $(I, a, G, b)$ are decided by the intrinsic characteristics of initiation and detonation. The constants of ignition $I$ and growth $G$ are set to $3.177 \times 10^{8} \mathrm{~s}^{-1}$ and $0.7045 \mathrm{~s}^{-1} \mathrm{~Pa}^{-b}$, respectively. The pressure sensitivity of the explosive is 0.7 , and the compression sensitivity is 4 [16].

\section{Experimental validations}

\subsection{Slow cook-off test}

To show validity and applicability of the extracted kinetic scheme to a practical thermal experiment, the following governing equations are considered:

$$
\begin{gathered}
\rho C \frac{d T}{d t}=k\left(\frac{\partial^{2} T}{\partial x^{2}}+\frac{\partial^{2} T}{\partial y^{2}}\right)-\frac{d \lambda}{d t} Q \\
\frac{d \lambda}{d t}=A_{\lambda} \exp \left(\frac{-E_{\lambda}}{R T}\right)
\end{gathered}
$$

Here $\rho, C$ and $k$ are density, specific heat and thermal conductivity respectively. In this experiment, the energetic materials do not 'flow' during the constant heating process. Thus mass and momentum are unchanged. The kinetic parameters of Fig. 3 is used in the rate equation. The cylindrical $2^{\text {nd }}$ order central difference scheme is used for the spatial discretization, and a $3^{\text {rd }}$ order RungeKutta method is used for time integration.

The schematic of the simulation is depicted in Fig. 5. The inside of cylindrical steel case is filled with the aluminized RDX. The temperature of the charge is measured at the center. The thickness of the steel casing is $0.4 \mathrm{~cm}$. The width and length of the charge are $4.5 \mathrm{~cm}$ and $20.0 \mathrm{~cm}$, respectively. The initial temperature of aluminized RDX is $30.8^{\circ} \mathrm{C}$ and then it is heated to $108^{\circ} \mathrm{C}$ within 1 hour. The heater temperature is maintained at $108^{\circ} \mathrm{C}$ for 7 hours to allow temperature to equilibrate before the final runaway to occur. Then the heating rate of $3.3^{\circ} \mathrm{C} /$ hour is used until explosion is witnessed.

The temperature histories are plotted in Fig. 6, and corresponding explosion time and temperature are listed in Table 1. A very good agreement between experiment and simulation by using the present chemical kinetics is obtained.

\subsection{Shock initiation test}

Another validation of the model is pursued using the pressure chamber test. A spherical charge of $3.5 \mathrm{~cm}$ radius with $1.78 \mathrm{~g} / \mathrm{cc}$ density is placed in a chamber maintained at 1 bar and detonated. Two pressure gauges are placed at two radial distances at $1.6 \mathrm{~m}$ and $1.9 \mathrm{~m}$ from the initial position of a charge. The purpose of the test was to obtain a primary detonation signal (pressure) followed by the sequent pressure peak representative of the aluminum afterburning. In order to 
simulate both chemical processes of detonation and aerobic reaction of aluminum, we use a multi-ignition reaction scheme for combined RDX and aluminum.

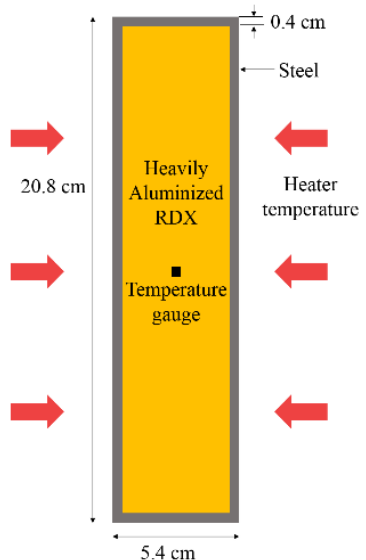

Figure 5: Schematic of thermal decomposition test.

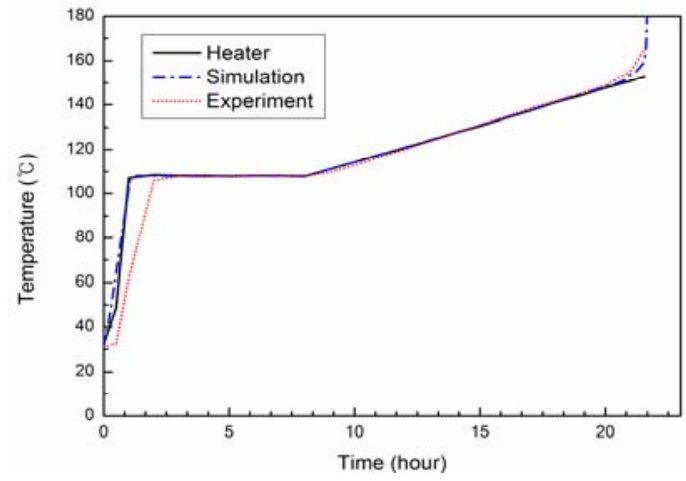

Figure 6: Temperature histories of slow cookoff test.

Table 1: Comparison of explosion time and temperature between experiment and simulation.

\begin{tabular}{ccc}
\hline \hline & Explosion time & Explosion temperature \\
\hline Experiment & $21.55 \mathrm{hrs}$ & $165.3^{\circ} \mathrm{C}$ \\
Prediction & $21.61 \mathrm{hrs}$ & $165.6^{\circ} \mathrm{C}$ \\
\hline
\end{tabular}

We assume that none of the aluminum particles reacted during the detonation of RDX. The afterburning of aluminum is modelled following the work of [17], which was originally applied for aluminum combustion in a gas phase. The aluminum ignition mechanism at high pressure condition is utilized to predict the afterburning of a heavily aluminized RDX.

A two-step Arrhenius-type mechanism for modeling the subsequent burning of aluminum that follows a detonation of RDX is

$$
\begin{aligned}
& \quad T>2000 \mathrm{~K}, \lambda_{A l}>0.01, \\
& \quad \frac{d \lambda_{A l}}{d t}=A\left(\rho_{0}\left(1-\lambda_{A l}\right)\right) d_{p}{ }^{2} \exp \left(-\frac{E_{a}}{R T}\right),\left(E_{a}=22.8 \mathrm{Kcal} / \mathrm{mol}, A=1 \times 10^{-2}\right) \\
& T<2000 \mathrm{~K}, \lambda_{A l} \geq 0.01, \\
& \frac{d \lambda_{A l}}{d t}=A\left(\rho_{0}\left(1-\lambda_{A l}\right)\right) \exp \left(-\frac{E_{a}}{R T}\right),\left(E_{a}=60 \mathrm{Kcal} / \mathrm{mol}, A=6.25 \times 10^{10}\right)
\end{aligned}
$$




$$
\begin{aligned}
& \rho_{A l_{0}}=a \rho_{0}, \quad \rho_{A l}=\left(1-\lambda_{A l}\right) \rho_{A l_{0}} \\
& \lambda_{T}=a \lambda_{A l}+b \lambda_{R D X}
\end{aligned}
$$

where $d_{p}$ or the particle diameter is $5 \mu \mathrm{m}$, while all other constants are referred from [17]. $\lambda_{A l}$ is the final product mass fraction of aluminum. $A$ is the preexponential factor, and $E_{a}$ is the activation energy. $\rho_{\text {Alo }}$ is the initial density of aluminum in the mixture, and $\rho_{\mathrm{Al}}$ is the calculated density of unreacted aluminum. $a$ is 0.35 for aluminum and $b$ is 0.65 for combined RDX/binder, both of which represent the initial mass percent of the mixture. Then $\lambda_{\mathrm{T}}$ represents total final product mass fraction. Also, the heat release due to aluminum reaction is $\dot{w}_{A l} q_{A l}$ which is added to the energy source $S$ of Eq. (21), where $\dot{w}_{A l}$ is aluminum reaction rate and $q_{\mathrm{Al}}$ is the enthalpy of reaction of aluminum $(85000 \mathrm{~kJ} / \mathrm{kg})$ [17].

As for the equation of state (EOS) of gas phase aluminum, we adapt the Noble-Abel equation of the form

$$
P_{A l}=\frac{a \lambda_{A l} \rho R T}{1-n A_{n}},\left(n A_{n}: \sim 0.75\right)
$$

where $R$ denotes gas constant, $n$ is the number of moles per unit volume, and $A_{n}$ is an empirical constant. Also, total pressure is defined as

$$
P_{\text {Total }}=\left(1-\lambda_{R D X}\right) P_{\text {unreacted }}+\lambda_{R D X} P_{\text {reacted }}+\lambda_{A l} P_{A l}
$$

Figure 7(a) shows different gauges along the radial distance from a charge center, and Figure 7(b) shows a timed pressure image of the chamber test. Aluminum is ignited at $650 \mu \mathrm{s}$ behind the detonated RDX gases. The burnt aluminum mass propagates toward the center as well as radially outward, because the fresh aluminum fuel is distributed within the radius of the hot product gas of RDX. Both primary peak of RDX and secondary peak of the afterburning are shown in the simulation.

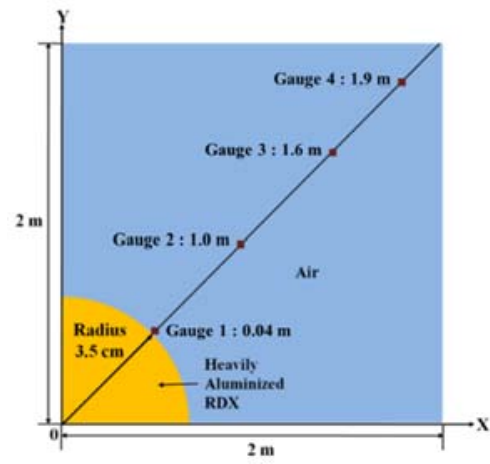

(a)

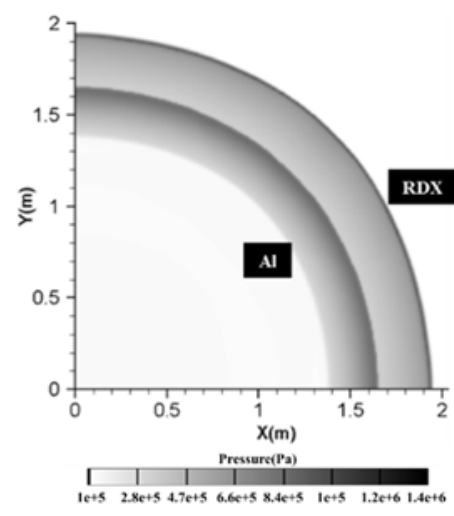

(b)

Figure 7: (a) Schematic of a charge detonation simulation and (b) calculated pressure of detonated RDX followed by aluminum afterburning at $1100 \mu \mathrm{s}$. 
Figure 8 shows a comparison of pressures, calculated and measured, at gauge locations 1.6 and 1.9 meters. Calculated RDX peak slightly over-predicts the measurement while the subsequent aluminum peak agrees with the measurement. From these figures, the primary wave average velocity is $1730 \mathrm{~m} / \mathrm{s}$ and the subsequent afterburning velocity is $410 \mathrm{~m} / \mathrm{s}$ as estimated at gauge $1.9 \mathrm{~m}$. One fourth of the RDX detonation wave speed approximates the average velocity of the afterburning of aluminum.

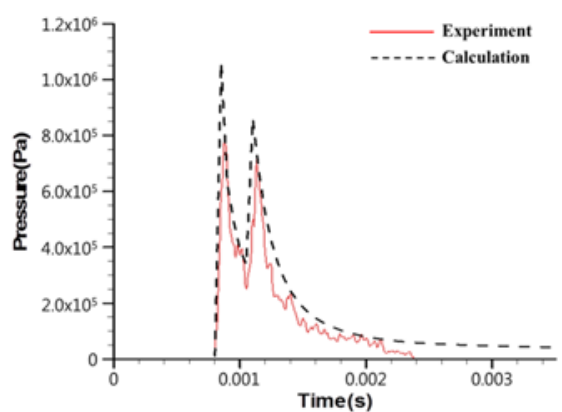

(a)

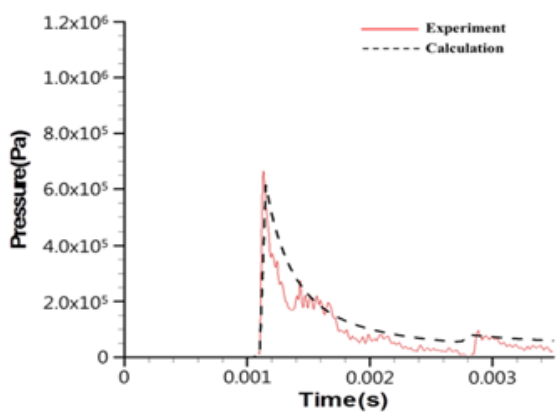

(b)

Figure 8: Comparison of pressure calculated and measured (a) at $1.6 \mathrm{~m}$ and (b) at $1.9 \mathrm{~m}$ gauge locations.

\section{Conclusions}

We present a set of chemical kinetics aimed at reliably predicting the response of heavily aluminized RDX subjected to i) thermal and ii) mechanical stimuli. Relevant experiments are conducted in order to validate the proposed reaction models. The method of extracting the necessary kinetics is general and thus additional propellants and high explosives can be considered for realistic hydrodynamic simulations of specific interests.

\section{Acknowledgement}

This work was supported by ADD contracted through the Institute of Advanced Aerospace Technology at Seoul National University.

\section{References}

[1] Melius, C. F., "Thermochemical Modeling: II. Application to Ignition and Combustion of Energetic Materials," Chemistry and Physics of Energetic Materials, Vol. 309, 1990, pp. 51-78 
[2] Burnham, A. K., Weese, R. K., Wardell, J. F., Tran, T. D., Wemhoff, A. P., Koerner, J. G., and Maienschein, J. L., "Can thermal analysis reliably predict thermal cookoff behavior," 13th International Detonation Symposium, Norfolk, VA, USA, July, 2006

[3] Tarver, C. M., and Tran, T. D., "Thermal decomposition models for HMX-based plastic bonded explosives," Combustion and Flame, Vol. 137, 2004, pp. 50-62

[4] Yoh, J. J., McClelland, M. A., Maienschein, J. L., Wardell, J. F., and Tarver, C. M., "Simulating thermal explosion of cyclotrimethylenetrinitramine-based explosives: Model comparison with experiment," J. Appl. Phys. Vol. 97, 2005, 083504

[5] Yoh, J. J., McClelland, M. A., Maienschein, J. L., Nichols, A. L., and Tarver, C. M., "Simulating thermal explosion of octahydrotetranitrotetrazine-based explosives: Model comparison with experiment," J. Appl. Phys. Vol. 100, 2006, 073515

[6] Friedman, H. L., "Kinetics of Thermal Degradation of Char-Forming Plastics from Thermogravimetry. Application to a Phenolic Plastic," Journal of Polymer Science, Vol. 6, 1963, pp. 183-195

[7] Vyazovkin, S., "Modification of the Integral Isoconversional Method to Account for Variation in the Activation Energy," Journal of Computational Chemistry, Vol. 22, No. 2, 2001, pp. 178-183

[8] Roduit, B., Borgeat, C., Berger, B., Folly, P., Andres, H., Schadeli, U., and Vogelsanger, B., Up-scaling of dsc data of high energetic materials. Journal of Thermal Analysis and calorimetry, Vol. 85, 2006, pp. 195-202

[9] Roduit, B., Folly, P., Berger, B., Mathieu, J., Sarbach, A., Andres, H., Ramin, M., and Vogelsanger, Advanced kinetics-based simulation of time to maximum rate under adiabatic conditions. Journal of Thermal Analysis and Calorimetry, Vol. 93, 2008, pp. 153-161

[10] Long, G. T., Vyazovkin, S., Brems, B. A., and Wight, C. A., "Competitive Vaporization and Decomposition of Liquid RDX," Journal of Physical Chemistry B, Vol. 104, 2000, pp. 2570-2574

[11] Lee, E. L. and Tarver, C. M. "Phenomenological model of shock initiation in heterogeneous explosives", Physics of Fluids, Vol. 23, No. 12, 1980, pp. 2362-2372

[12] Souers, P. C., Anderson, S., Mercer, J., McGuire, E., and Vitello, P., "JWL++: A Simple Reactive Flow Code Package for Detonation", Propellants, Explosives, Pyrotechnics, Vol. 25, No. 2, 2000, pp. 54-58

[13] Tarver, C. M., Hallquist, J. O., and Erickson, L. M., "Modeling short pulse duration shock initiation of solid explosives", Proceedings of the 8th Symposium on Detonation, 1985, pp. 951-961

[14] Guilkey, J. E., Harman, T. B., and Banerjee, B., "An Eulerian-Lagrangian Approach for Simulating Explosions of Energetic Devices", Computers and Structures, Vol. 85, 2007, pp. 660-674

[15] Lee, E. L., Hornig, H. C., and Kury, J. W., "Adiabatic Expansion of High Explosive Detonation Products”, LLNL, UCRL-50422, 1968, pp. 1-21 
[16] Kim, B., Park, J., Lee, K.-C., and Yoh, J. J., "A reactive flow model for heavily aluminized cyclotrimethylene-trinitramine," J. Appl. Phys. Vol. 116, 2014, 023512

[17] Kuhl, A. L., Bell, J. B., and Beckner, V. E., "Heterogeneous continuum model of aluminum particle combustion in explosions," Combustion, Explosion, and Shock Waves, Vol. 46, No. 4, 2010, pp. 433-448 